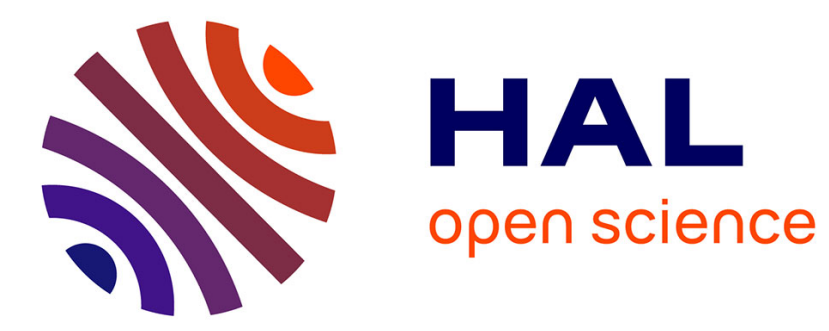

\title{
IDA-PBC for LTI dynamics under input delays: a reduction approach
}

\author{
Mattia Mattioni, Salvatore Monaco, Dorothée Normand-Cyrot
}

\section{To cite this version:}

Mattia Mattioni, Salvatore Monaco, Dorothée Normand-Cyrot. IDA-PBC for LTI dynamics under input delays: a reduction approach. IEEE Control Systems Letters, 2020, 10.1109/LCSYS.2020.3040076 . hal-03020691

\section{HAL Id: hal-03020691 https://hal.science/hal-03020691}

Submitted on 24 Nov 2020

HAL is a multi-disciplinary open access archive for the deposit and dissemination of scientific research documents, whether they are published or not. The documents may come from teaching and research institutions in France or abroad, or from public or private research centers.
L'archive ouverte pluridisciplinaire HAL, est destinée au dépôt et à la diffusion de documents scientifiques de niveau recherche, publiés ou non, émanant des établissements d'enseignement et de recherche français ou étrangers, des laboratoires publics ou privés. 


\title{
IDA-PBC for LTI dynamics under input delays: a reduction approach
}

\author{
Mattia Mattioni $^{1}$, Salvatore Monaco ${ }^{1}$ and Dorothée Normand-Cyrot ${ }^{2}$
}

\begin{abstract}
In this paper, the problem of stabilizing linear port-controlled Hamiltonian dynamics through interconnection and damping assignment in presence of input delays is considered. The contribution exploits the reduction approach allowing to reveal and shape the energy properties of the time-delay dynamics. Performances are illustrated on a simple mechanical system.
\end{abstract}

Index Terms-Linear systems, Delay systems, Energy systems.

\section{INTRODUCTION}

Passivity-based control (PBC) of dynamical systems has been shown to offer a natural and powerful framework for controlling dynamic systems by exploiting their physical properties [1], [2]. Basically, when considering stabilization at the origin, $\mathrm{PBC}$ is aimed at injecting energy back through feedback so to modify and exploit the natural dissipation of the plant [3]. When considering port-controlled Hamiltonian systems [4], [5], Interconnection and Damping Assignment (IDA, [6]) has been introduced to stabilize the system at some desired new equilibria through passivation: first the energy of the system is reshaped; then damping is injected to ensure asymptotic convergence to the desired equilibrium.

If on one side, PBC represents a powerful control approach at large and several extensions have been proposed in the literature [7], [8], a very few works are devoted to the investigation and exploitation of passivity in presence of time delays (e.g., [9]-[13]). When considering nonlinear dynamics affected by discrete input delays of length $\tau$, a new solution concerning passivity-based control for stabilization at the origin has been proposed in [14] through reduction [15][17]. The main idea is to construct a new dynamics (the reduced dynamics) that is free of delays and equivalent, in terms of stabilizability, to the original delayed one. Such a dynamics preserves the drift of the retarded one but exhibits a transformed control vector field explicitly parameterized by the delay. Then, passivity-based arguments for stabilization at the origin can be fruitfully applied.

Exploiting the approach proposed in [14], the contribution of this paper concerns stabilization of linear time-invariant (LTI) port-controlled Hamiltonian dynamics under input delay at a desired equilibrium through IDA-PBC; namely, stabilization is performed by assigning and shaping the energy

Supported by Sapienza Università di Roma (Progetti di Ateneo 2018Piccoli progetti RP11816436325B63).

${ }^{1}$ Dipartimento di Ingegneria Informatica, Automatica e Gestionale $A$. Ruberti (Sapienza University of Rome); Via Ariosto 25, 00185 Rome, Italy \{ mattia.mattioni, salvatore.monaco\} @uniromal.it.

${ }^{2}$ Laboratoire de Signaux et Systèmes (L2S, CNRS); 3, Rue Joliot Curie, 91192, Gif-sur-Yvette, France dorothee.normand-cyrot @centralesupelec.fr dissipation of the time-delay closed-loop system. Assuming the existence of a solution for the delay-free case, we show first how to define and compute a reduced dynamics which preserves the Hamiltonian structure of the original retarded dynamics together with its dissipation. Then, an IDA-PBC feedback designed over the reduced dynamics is shown to asymptotically stabilize the retarded original dynamics at the desired equilibrium. The designed feedback assigns, to the reduced dynamics, the same Hamiltonian structure as the one assigned to the delay-free feedback system. As an important consequence of this choice, it is shown that the resulting delayed feedback control system possesses a new portHamiltonian structure which is directly parameterized by the delay and which recovers the delay-free one as $\tau$ falls to zero. Several extensions are briefly sketched. A simple example is exploited to illustrate the result and perform comparisons with respect to classical prediction-based control laws.

The remainder of this paper is organized as follows. In Section $[\mathrm{I}$. the problem is settled by providing recalls on standard IDA-PBC and reduction-based control. In Section III. the main results are given with some sketches on the extension to further classes of time-delay systems. In Section $[\mathrm{IV}$ a simple mechanical LTI system is worked out and simulations show the performances. Final remarks and perspectives conclude the paper.

Notations. $\mathbb{R}$ and $\mathbb{N}$ denote the set of real and natural numbers including 0 . For any vector $z \in \mathbb{R}^{n},\|z\|$ and $z^{\top}$ define respectively the norm and transpose of $z$. Given a full rank matrix $B \in \operatorname{mat}_{\mathbb{R}}(n, m)$ with $n>m$, $B^{\dagger}=\left(B^{\top} B\right)^{-1} B^{\top}$ denotes the pseudoinverse, while $B^{\perp}$ its orthogonal complement verifying $B^{\perp} B=0$. Also, $\operatorname{ker}\{B\}$ denotes the nullspace of $B$. Given $R \in \operatorname{Mat}_{\mathbb{R}}(n, n)$ and $z \in$ $\mathbb{R}^{n}$, the weighted square norm is defined as $\|z\|_{R}^{2}:=z^{\top} R z$. I denotes the identity matrix of suitable dimension. Given a twice continuously differentiable function $S(\cdot): \mathbb{R}^{n} \rightarrow \mathbb{R}$, $\nabla S(\cdot)$ represents its gradient vector.

\section{PReliminaries AND PROBlem Statement}

In this section, recalls on reduction for LTI dynamics dynamics [14], [18] are provided together with standard results on IDA-PBC for delay-free port-controlled Hamiltonian (pcH) dynamics [19].

\section{A. The reduction approach for LTI dynamics}

Consider a retarded LTI dynamics of the form

$$
\dot{x}(t)=A x(t)+B u(t-\tau)
$$

with $x \in \mathbb{R}^{n}, u \in \mathbb{R}^{m}, m \leq n$ and $\tau>0$ a constant and known time delay. For simplicity, we assume $B$ of full rank 
and $u(t)=0$ for $t \in[-\tau, 0)$. As firstly proposed in [18] with extension to the nonlinear context in [14], the problem of controlling (1) can be set over a simplified dynamics which is free of delay. To this end, we introduce the socalled reduction variable

$$
\eta(t)=x(t)+\int_{t-\tau}^{t} e^{(t-\tau-\ell) A} B u(\ell) \mathrm{d} \ell
$$

with initial condition $\eta_{0}=x_{0}$, which can be easily verified to evolve with the reduced dynamics

$$
\dot{\eta}(t)=A \eta(t)+B_{\tau} u(t)
$$

where $B_{\tau}=e^{-\tau A} B$. It results that the reduced dynamics (3) preserves the same drift as (1) with constant control vector field parameterized by $\tau, B_{\tau}$. Accordingly, from [18], all feedbacks stabilizing (3) ensure stabilization of (1). Moreover, by construction, all properties of (1) are preserved by reduction as, for instance, passivity. This last aspect is clarified by the following statement recalled from [14].

Proposition 2.1: Let the delay-free (with $\tau=0$ ) dynamics associated to 11 be passive with output $y=C x, C=B^{\top} P$, and storage function $H(x)=x^{\top} P x, P \succ 0$; then, the following holds true:

1) the reduced dynamics (3) is passive with output $y_{\tau}(t)=C_{\tau} \eta(t)$ with $C_{\tau}=B_{\tau}^{\top} P$ and the same storage function $H(\eta)=\eta^{\top} P \eta$

2 ) if for $\tau=0$, the delay-free dynamics associated to (1) is Zero-State-Detectable (ZSD), then the reduced dynamics (3) with output $y_{\tau}(t)=C_{\tau} \eta(t)$ is ZSD;

3 ) the retarded dynamics (1) is passive with output

$$
y_{\tau}\left(x(t), u_{t}\right)=C_{\tau} x(t)+\int_{t-\tau}^{t} C_{\tau} e^{(t-\tau-\ell) A} B u(\ell) \mathrm{d} \ell
$$

with $u_{t}=u_{t}(\theta)=u(t+\theta)$ for $\theta \in[-\tau, 0)$ and storage functional

$$
H_{\tau}\left(x(t), u_{t}\right)=\left\|x(t)+\int_{t-\tau}^{t} e^{(t-\tau-\ell) A} B u(\ell) \mathrm{d} \ell\right\|_{P}^{2} .
$$

Remark 2.1: In case of discrete and uniform input delay, the reduction variable (2) rewrites as $\eta(t)=e^{-\tau A} x(t+\tau)$ for $x(t+\tau)=e^{\tau A} x(t)+\int_{t-\tau}^{t} e^{A(t-\ell)} u(\ell) \mathrm{d} \ell$. Accordingly, reduction can be interpreted as the prediction of the state brought backward in time through $e^{-\tau A}$ which defines the free evolution.

\section{B. IDA-PBC for LTI pcH systems}

Consider a delay-free $\mathrm{pcH}$ system of the form

$$
\dot{x}(t)=(J-R) \nabla H(x(t))+B u(t)
$$

verifying, by construction, for all $t \geq 0$ the energy-balance equality

$$
H(x(t))-H\left(x_{0}\right)=\int_{0}^{t} y^{\top}(\ell) u(\ell) \mathrm{d} \ell-w(t)
$$

where, for some $P \succ 0, w(t) \geq 0$ and $y(t)=B^{\top} P x(t)$ denote the dissipated energy and the corresponding passive conjugate output, respectively and $H(x)=x^{\top} P x$ is the socalled Hamiltonian.

Let $x_{\star} \in \operatorname{ker}\left\{B^{\perp} A\right\}$ be a desired equilibrium to stabilize and assign via IDA-PBC with $v$ an exogenous input signal; one seeks for a control law $u(t)=u_{\mathrm{es}}(t)+u_{\mathrm{di}}(t)+v(t)$ with: $u_{\mathrm{es}}$ being the energy shaping component modifying the total energy function to assign the desired equilibrium $x_{\star}$ and $u_{\mathrm{di}}$ the damping injection component achieving asymptotic stability of the aforementioned point. The problem is reduced to the computation of matrices $F, F_{\star}, J_{d}=-J_{d}^{\top}, R_{d}=$ $R_{d}^{\top} \succeq 0$ and $P_{d}=P_{d}^{\top} \succ 0$ solutions to the so-called matching equation

$$
\begin{aligned}
& \left(J_{d}-R_{d}\right) \nabla H_{d}(x) \\
& -(J-R) \nabla H(x)-B\left(F x+F_{\star} x_{\star}\right)=0
\end{aligned}
$$

so that (4) rewrites, in closed loop, as the $\mathrm{pcH}$ system

$$
\dot{x}(t)=\left(J_{d}-R_{d}\right) \nabla H_{d}(x(t))+B v(t)
$$

with new Hamiltonian $H_{d}(x)=\left(x-x_{\star}\right)^{\top} P_{d}\left(x-x_{\star}\right)$ and control $u=F x+F_{\star} x_{\star}+v$. As a consequence, one gets that the closed-loop $\mathrm{pcH}$ system is passive with respect to the new output $y_{d}=B^{\top} P_{d}\left(x-x_{\star}\right)$, energy balance equality

$$
H_{d}(x(t))-H_{d}\left(x_{0}\right)=\int_{0}^{t} y_{d}^{\top}(\ell) v(\ell) \mathrm{d} \ell-w_{d}(t)
$$

and $x_{\star}$ as a new (asymptotically) stable equilibrium. As wellknown [19], in the LTI case the matching equation (5) is equivalent to the matrix equalities

$$
\begin{aligned}
& \left(J_{d}-R_{d}\right) P_{d}-(J-R) P-B F=0 \\
& -\left(J_{d}-R_{d}\right) P_{d}+B F_{\star}=0
\end{aligned}
$$

with $P_{d}, J_{d}$ and $R_{d}$ verifying the so-called matching condition

$$
B^{\perp}\left[\left(J_{d}-R_{d}\right) P_{d}-(J-R) P\right]=0
$$

that is necessary for solvability of the problem. Under (6) the control matrices are given by

$$
\begin{aligned}
F & =B^{\dagger}\left[\left(J_{d}-R_{d}\right) P_{d}-(J-R) P\right] \\
F_{\star} & =-B^{\dagger}\left(J_{d}-R_{d}\right) P_{d} .
\end{aligned}
$$

Remark 2.2: Typically, one usually sets the dissipation matrix as $R_{d}=\kappa B B^{\top}$ with $\kappa>0$, that is the one resulting from damping injection from the new passive output $y_{d}=B^{\top} P_{d}\left(x-x_{\star}\right)$.

\section{Problem formulation and outline of the results}

From now on, we assume that (1) is a port-Hamiltonian dynamics of the form

$$
\dot{x}(t)=(J-R) \nabla H(x(t))+B u(t-\tau)
$$

with $J=-J^{\top}, R=R^{\top} \succeq 0, H(x)=x^{\top} P x, P \succ 0$ and $A=(J-R) P$. In addition, we refer to (4) as the corresponding delay-free system deduced from (8) when $\tau=$ 0 under the following standing assumption which is typical of the time-delay context. 
Assumption 1: There exists a stabilizing IDA-PBC solution for the delay-free dynamics (4); namely, there exist $J_{d}=-J_{d}^{\top}, R_{d}=R_{d}^{\top} \succeq 0, H_{d}(x)=\left(x-x_{\star}\right)^{\top} P_{d}\left(x-x_{\star}\right)$, $P_{d}=P_{d}^{\top} \succ 0$ solution to the matching equality (5) with the feedback $u=F x+F_{\star} x_{\star}+v$ with $F$ and $F_{\star}$ in (7), which asymptotically stabilizes $x_{\star} \in \operatorname{ker}\left\{B^{\perp} A\right\}$.

In the following, we seek to assign a desired equilibrium $x_{\star}$ to the retarded system (8) by assigning a desired energy behavior in closed loop despite the effect of the delay $\tau>0$. In particular, exploiting reduction arguments and Assumption 1. we show that a suitable IDA-PBC problem can be set and solved over the reduced model to fulfill the specification over the delayed $\mathrm{pcH}$ dynamics (8). In this sense, we underline that Assumption 1 is not restrictive as IDA-PBC is generally relying on the free evolution of the system rather than on its forced component. The following items outline the design procedure:

1) construct the reduced model associated to (8), that is

$$
\dot{\eta}(t)=(J-R) \nabla H(\eta(t))+B_{\tau} u(t) ;
$$

2) set an IDA-PBC control problem over (9) to assign and stabilize a suitably defined reduced equilibrium $\eta_{\star} \in \operatorname{ker}\left\{B_{\tau}^{\perp} P\right\}$ uniquely corresponding to $x_{\star} \in$ $\operatorname{ker}\left\{B^{\perp} A\right\}$; i.e. compute $F_{\tau}, F_{\tau_{\star}}, J_{d, \tau}=-J_{d, \tau}^{\top}$, $R_{d, \tau}=R_{d, \tau}^{\top} \succeq 0$ and $P_{d, \tau}=P_{d, \tau}^{\top} \succ 0$ solutions to the reduced matching equation

$$
\begin{aligned}
& \left(J_{d, \tau}-R_{d, \tau}\right) \nabla H_{d, \tau}(\eta) \\
& \left.-(J-R) \nabla H(\eta)-B_{\tau}\left(F_{\tau} \eta+F_{\tau_{\star}} \eta_{\star}\right)\right]=0 .
\end{aligned}
$$

The closed-loop reduced-model gets the form

$$
\dot{\eta}(t)=\left(J_{d, \tau}-R_{d, \tau}\right) \nabla H_{d, \tau}(\eta(t))+B_{\tau} v(t)
$$

with reduced Hamiltonian $H_{d, \tau}(\eta(t))=\left\|\eta-\eta_{\star}\right\|_{P_{d, \tau}}^{2}$ and $u(t)=F_{\tau} \eta(t)+F_{\tau_{\star}} \eta_{\star}+v$.

Accordingly, the contributions of the paper are given below:

1) under Assumption 1 a solution to the set IDA-PBC control problem is shown to exist for all $\tau>0$ for the reduced model (9); the defined feedback stabilizes the suitably defined reduced equilibrium $\eta_{\star} \in \operatorname{ker}\left\{B_{\tau}^{\perp} A\right\}$ and preserves the same Hamiltonian, damping and interconnection matrices as in the delay-free case (Proposition 3.1);

2) the controller designed on the reduced model assigns and stabilizes the desired equilibrium $x_{\star} \in \operatorname{ker}\left\{B^{\perp} A\right\}$ of the original delayed dynamics (8) by assigning a new Hamiltonian structure (Theorem 3.1.

\section{REDUCTION-BASED IDA-PBC}

\section{A. Main result}

As mentioned above, the objective is to regulate the state of the time-delay pcH dynamics (8) to a desired admissible equilibrium $x_{\star} \in \operatorname{ker}\left\{B^{\perp} A\right\}$ through IDA-PBC over the reduced dynamics (9). To this end, it is first necessary to characterize the correspondence among admissible equilibria $x_{\star} \in \operatorname{ker}\left\{B^{\perp} A\right\}$ for $(8)$ and admissible equilibria $\eta_{\star} \in$
$\operatorname{ker}\left\{B_{\tau}^{\perp} P\right\}$ for the reduced $\mathrm{pcH}$ dynamics 9 . The result below serves this purpose.

Lemma 3.1: For all fixed $\tau>0$, each admissible equilibrium $x_{\star} \in \operatorname{ker}\left\{B^{\perp} A\right\}$ of the original retarded system 8 corresponds to $\eta_{\star}=e^{-\tau A} x_{\star}$ as admissible equilibrium of the reduced dynamics 9 .

Proof: The result is proved by fixing $B_{\tau}^{\perp}=B^{\perp} e^{\tau A}$ with $A=(J-R) P$ and the (constant) feedback $u_{\tau_{\star}}=$ $-B_{\tau}^{\dagger} A \eta_{\star}$ assigning $\eta_{\star}=e^{-\tau A} x_{\star}$. Accordingly, by definition of reduction in (2), one gets that for all $t \geq 0$

$$
x(t)=e^{-\tau A} x_{\star}+\int_{t-\tau}^{t} e^{A(t-\ell)} \mathrm{d} \ell B_{\tau} B_{\tau}^{\dagger} A e^{-\tau A} x_{\star}
$$

so that one needs to prove that, for all $t \geq 0$

$$
x_{\star}=e^{-\tau A} x_{\star}+\int_{t-\tau}^{t} e^{A(t-\ell)} \mathrm{d} \ell B_{\tau} B_{\tau}^{\dagger} A e^{-\tau A} x_{\star}
$$

and thus the quality

$$
\left(\mathrm{I}-e^{-\tau A}\right) x_{\star}=\int_{t-\tau}^{t} e^{A(t-\ell)} \mathrm{d} \ell B_{\tau} B_{\tau}^{\dagger} A e^{-\tau A} x_{\star} .
$$

By exploiting the definition of pseudo-inverse and the fact that $e^{-\tau A} x_{\star} \in \operatorname{ker}\left\{B_{\tau}^{\perp} A\right\}$ one gets

$$
\begin{aligned}
\int_{t-\tau}^{t} e^{A(t-\ell)} \mathrm{d} \ell B_{\tau} B_{\tau}^{\dagger} A e^{-\tau A} x_{\star} & =\int_{t-\tau}^{t} e^{A(t-\ell)} \mathrm{d} \ell A e^{-\tau A} x_{\star} \\
& =\int_{0}^{\tau} e^{A s} \mathrm{~d} s A e^{-\tau A} x_{\star} \\
& =\left(\mathrm{I}-e^{-\tau A}\right) x_{\star}
\end{aligned}
$$

so getting the result.

We are now ready to prove the following result.

Proposition 3.1: Consider the pcH dynamics (8) affected by a discrete input delay of length $\tau>0$ with $x_{\star} \in$ $\operatorname{ker}\left\{B^{\perp} A\right\}$ a desired equilibrium to be assigned and stabilized. Let (8) verify Assumption 1 with matrices $J_{d}=-J_{d}^{\top}$, $R_{d}=R_{d}^{\top} \succeq 0$ and $P_{d}=P_{d}^{\top} \succ 0$. Let (2) be the reduction variable associated to (8) with reduced model (9) and equilibrium $\eta_{\star}=e^{-\tau A} x_{\star}$ to be assigned. Then, the reduced matching equation 10 is solved by $J_{d, \tau}=J_{d}$, $R_{d, \tau}=R_{d}, H_{d, \tau}(\eta)=H_{d}(\eta)=\left(\eta-\eta_{\star}\right)^{\top} P_{d}\left(\eta-\eta_{\star}\right)$ and the feedback law $u(t)=F_{\tau} \eta(t)+F_{\tau_{\star}} \eta_{\star}+v(t)$ with

$$
\begin{aligned}
F_{\tau} & =B_{\tau}^{\dagger}\left[\left(J_{d}-R_{d}\right) P_{d}-(J-R) P\right] \\
F_{\tau_{\star}} & =-B_{\tau}^{\dagger}\left(J_{d}-R_{d}\right) P_{d}
\end{aligned}
$$

making the closed-loop reduced model of the form

$$
\dot{\eta}(t)=\left(J_{d}-R_{d}\right) \nabla H_{d}(\eta(t))+B_{\tau} v(t) .
$$

In addition, the closed-loop reduced dynamics (12) with output $y_{d, \tau}(t)=B_{\tau}^{\top} P_{d}\left(\eta(t)-\eta_{\star}\right)$ is passive.

Proof: The proof works out by showing that $J_{d}, R_{d}$ and $P_{d}$ solve the reduced matching condition

$$
B_{\tau}^{\perp}\left[\left(J_{d, \tau}-R_{d, \tau}\right) P_{d, \tau}-(J-R) P\right]=0 .
$$

To this end and with a slight abuse of notation, setting $B_{\tau}^{\perp}=B^{\perp} e^{\tau A}$ with $A=(J-R) P$ and $A_{d}=\left(J_{d}-R_{d}\right) P_{d}$, 
one gets $B_{\tau}^{\perp}\left[A_{d}-A\right]=B^{\perp} e^{\tau A}\left[A_{d}-A\right]$. Rewriting now $e^{\tau A}=I+\sum_{i>0} \frac{\tau^{i}}{i !} A^{i}$ and exploiting, by Assumption 1 . the equality $B^{\perp} A=B^{\perp} A_{d}$ it is a matter of computations to verify that $B^{\perp} e^{\tau A} A_{d}=B^{\perp} A_{d} e^{\tau A}$ for all $\tau>0$, that is $B^{\perp} A^{i} A_{d}=B^{\perp} A^{i+1}$ for all $i=0,1, \ldots$ This can be proved by induction assuming that this holds for $i \geq 0$. Accordingly, it is a matter of computations to check that the $i^{\text {th }}$ order equality rewrites as $B^{\perp} A^{i} A_{d}-B^{\perp} A^{i+1}=$ $B^{\perp} A_{d}^{i+1}-B^{\perp} A^{i+1}=B^{\perp}\left(A_{d}^{i}-A^{i}\right)\left(A_{d}+A\right)=0$ so that $B^{\perp} A^{i+1} A_{d}-B^{\perp} A^{i+2}=B^{\perp} A_{d}^{i+2}-B^{\perp} A^{i+2}=0$ and thus the result.

Remark 3.1: It is worth to note that the prediction-based feedback $u_{p}(t)=F x(t+\tau)+F_{\star} x_{\star}$ with $F, F_{\star}$ as in $(7)$ and $x(t+\tau)=e^{\tau A} \eta(t)$ solves the reduced matching condition (13) with $A_{d, \tau}=\left(J_{d, \tau}-R_{d, \tau}\right) P_{d, \tau}=e^{-\tau A}\left(J_{d}-R_{d}\right) P_{d} e^{\tau A}$. In particular, $J_{d, \tau}=e^{-\tau A} J_{d} e^{\tau A}, R_{d, \tau}=e^{-\tau A} R_{d} e^{\tau A}$ and $P_{d, \tau}=e^{-\tau A} P_{d} e^{\tau A}$ that is neither symmetric nor positive semidefinite in general. In addition, such prediction does not yield an IDA-PBC control under reduction as not ensuring a closed-loop pcH structure for $(9)$ for $J \neq 0$.

Remark 3.2: A further solution to the reduced matching equality (13) is provided by $P_{d, \tau}=P_{d}, J_{d, \tau}=J_{d}$ and $R_{d, \tau}=\kappa_{\tau} B_{\tau} B_{\tau}^{\top}$ with $\kappa_{\tau}>0$, that is the one resulting from performing damping injection over the new passivating reduced output $y_{\tau}=B_{\tau}^{\top} P_{d}\left(\eta-\eta_{\star}\right)$. Such a solution assigns to the closed-loop time-delay systems the damping matrix $\tilde{R}_{d, \tau}=R_{d}=B B^{\top}$.

Proposition 3.1 shows that, when IDA-PBC can be applied to stabilize a desired equilibrium $x_{\star} \in \operatorname{ker}\left\{B^{\perp} A\right\}$ for the delay-free $\mathrm{pcH}$ system (4), an IDA-PBC control can always be computed over the reduced model in presence of input delays. Although the structure of the closed-loop reduced model is the same as the delay-free one, the corresponding feedback laws are not. In addition, even more importantly, the reduction-based feedback assigns different $\mathrm{pcH}$ and energetic structures to the time-delay system (8) with respect to the delay-free case. Thus, the following question naturally arises: what is the structure that the reduction-based IDAPBC feedback assigns to the original retarded dynamics (8)? The answer is provided by the result below.

Theorem 3.1: Consider the pcH dynamics $(8)$ affected by a discrete input delay of length $\tau>0$ with $x_{\star} \in \operatorname{ker}\left\{B^{\perp} A\right\}$ a desired equilibrium to be assigned and stabilized via IDAPBC. Let 8 verify Assumption 1 with matrices $J_{d}=-J_{d}^{\top}$, $R_{d}=R_{d}^{\top} \succeq 0$ and $P_{d}=P_{d}^{\top} \succ 0$. Then, the reductionbased IDA-PBC feedback (11) makes the system (8) a portHamiltonian system of the form

$$
\dot{x}(t)=\left(\tilde{J}_{d}-\tilde{R}_{d}\right) \nabla \tilde{H}_{d}(x(t))+B v(t-\tau)
$$

with $\tilde{J}_{d}=e^{\tau A} J_{d} e^{\tau A^{\top}}=-\tilde{J}_{d}^{\top}, \tilde{R}_{d}=e^{\tau A} R_{d} e^{\tau A^{\top}}=\tilde{R}_{d}^{\top} \succeq$ $0, \tilde{H}_{d}(x)=\left(x-x_{\star}\right)^{\top} e^{-\tau A^{\top}} P_{d} e^{-\tau A}\left(x-x_{\star}\right)$ and asymptotically stable equilibrium at the desired $x_{\star} \in \operatorname{ker}\left\{B^{\perp} A\right\}$ with $A=(J-R) P$.

Proof: To investigate this latter aspect, we consider the delayed pcH dynamics (8) under the reduction-based IDAPBC feedback in Proposition 3.1 (computed for simplicity at $v=0)$

$$
\dot{x}(t)=A x(t)+B\left[B_{\tau}^{\dagger}\left(A_{d}-A\right) \eta(t-\tau)-B_{\tau}^{\dagger} A_{d} e^{-\tau A} x_{\star}\right]
$$

with $A=(J-R) P$ and $A_{d}=\left(J_{d}-R_{d}\right) P_{d}$. By exploiting Remark 2.1. one gets $\eta(t-\tau)=e^{-\tau A} x(t)$ so getting

$$
\begin{aligned}
\dot{x}(t) & =A x(t)+B B_{\tau}^{\dagger}\left[\left(A_{d}-A\right) e^{-\tau A} x(t)-A_{d} e^{-\tau A} x_{\star}\right] \\
& =A x(t)+e^{\tau A} e^{-\tau A} B B_{\tau}^{\dagger}\left[\left(A_{d}-A\right) e^{-\tau A} x(t)-A_{d} e^{-\tau A} x_{\star}\right] \\
& =A x(t)+e^{\tau A}\left[\left(A_{d}-A\right) e^{-\tau A} x(t)-A_{d} e^{-\tau A} x_{\star}\right] \\
& =e^{\tau A} A_{d} e^{-\tau A}\left(x(t)-x_{\star}\right) .
\end{aligned}
$$

The form above is a pcH dynamics with Hamiltonian $\tilde{H}_{d}(x)=\left(x-x_{\star}\right)^{\top} e^{-\tau A^{\top}} P_{d} e^{-\tau A}\left(x-x_{\star}\right)$ and new damping and interconnection matrices $\tilde{J}_{d}=e^{\tau A} J_{d} e^{\tau A^{\top}}=-\tilde{J}_{d}^{\top}$, $\tilde{R}_{d}=e^{\tau A} R_{d} e^{\tau A^{\top}}=\tilde{R}_{d}^{\top} \succeq 0$.

Remark 3.3: Contrarily to the prediction discussed in Remark 3.1. the proposed reduction-based feedback assigns a new structure to the retarded $\mathrm{pcH}$ dynamics (8) with new damping and interconnection matrices that are directly parameterized by $\tau$. However, as $\tau \rightarrow 0$ the proposed solution naturally recovers the delay-free one.

Remark 3.4: Along the lines of Proposition 2.1 and the results in [14], for $A_{d}=\left(J_{d}-R_{d}\right) P_{d}$ the time-delay closedloop system 14 is passive with respect to the output

$$
\begin{aligned}
y_{d, \tau}\left(x(t), v_{t}\right)= & B_{\tau}^{\top} P_{d}\left(x(t)-x_{\star}\right) \\
& +\int_{t-\tau}^{t} B_{\tau}^{\top} P_{d} e^{A_{d}(t-\tau-\ell)} v(\ell) \mathrm{d} \ell .
\end{aligned}
$$

\section{B. Further remarks and extensions}

1) An insight on robustness analysis: Contrarily to prediction-based feedback [20], reduction is well-known to be robust with respect to uncertainties in the knowledge of the delay. To this end, assume that $\tau \in\left(0, \tau_{n}\right]$ is unknown but bounded by a known and constant $\tau_{n}>0$. In this case, the $\mathrm{pcH}$ structure of the closed-loop feedback under reduction-based feedback is not preserved. Still stabilization of the desired equilibrium might still hold under $u(t)=$ $F_{\tau_{n}} \eta(t)+F_{\tau_{n_{\star}}} \eta_{\star}$ computed over the nominal reduction

$$
\eta(t)=x(t)+\int_{t-\tau_{n}}^{t} e^{A\left(t-\tau_{n}-\ell\right)} B u(\ell) \mathrm{d} \ell
$$

evolving with perturbed closed-loop reduced model

$$
\dot{\eta}(t)=\left(J_{d}-R_{d}\right) P_{d} \eta(t)+B F_{\tau_{n}}\left(\eta(t-\tau)-\eta\left(t-\tau_{n}\right)\right) .
$$

To investigate robustness of the closed-loop system (8) under the nominal reduction-based feedback it is hence enough to investigate robust stability of the perturbed reduced dynamics (16). To this end, as commented in Remark 2.2. one can set $R_{d}=\kappa B B^{\top}$ and look for $\kappa>0$ preserving stability of 16 and hence of $(8)$ in closed loop despite the delay mistmatch. This can be done exploiting standard Lyapunov-Krasovskii arguments [21] rewriting [16] as

$$
\dot{\eta}(t)=\left(J_{d}-R_{d}\right) P_{d} \eta(t)+B F_{\tau_{n}} \int_{t-\tau_{n}}^{t-\tau} \dot{\eta}(\ell) \mathrm{d} \ell
$$


with the functional

$$
\mathcal{V}\left(\eta_{t}\right)=H_{d}(\eta(t))+\frac{\varepsilon}{\tau_{n}} \int_{t-\tau_{n}}^{t} \int_{\ell}^{t}\|\dot{\eta}(s)\|^{2} \mathrm{~d} s \mathrm{~d} \ell, \quad \varepsilon>0 .
$$

Remark 3.5: As well known, prediction-based feedback suffers from initialization issues as, setting $z(t)=x(t+\tau)$, one gets $z_{0}=x(\tau)=e^{\tau A} x_{0}$. This is crucial when the delay is uncertain since the initial condition for prediction cannot be exactly fixed apriori. As underlined so far, this issue is overcome by the reduction approach yielding $\eta_{0}=x_{0}$.

2) Extensions to further classes of time-delay systems: The proposed result applies to dynamics affected by multichannel delays described by

$$
\dot{x}(t)=(J-R) \nabla H(x(t))+\sum_{i=1}^{m} b_{i} u_{i}\left(t-\tau_{i}\right)
$$

with $\tau_{i}>0$ for $i=1, \ldots, m$. As a matter of fact, one introduces the reduction and the reduced dynamics as

$$
\begin{aligned}
& \eta(t)=x(t)+\sum_{i=1}^{m} \int_{t-\tau_{i}}^{t} e^{\left(t-\tau_{i}-\ell\right) A} b_{i} u_{i}(\ell) \mathrm{d} \ell \\
& \dot{\eta}(t)=(J-R) \nabla H(\eta(t))+\sum_{i=1}^{m} e^{-\tau_{i} A} b_{i} u_{i}(t) .
\end{aligned}
$$

Also, the case of dynamics affected by distributed delays can be dealt using the proposed arguments. In particular, considering the time-delay $\mathrm{pcH}$ dynamics

$$
\dot{x}(t)=(J-R) \nabla H(x(t))+\int_{0}^{\tau} B(\ell) u(t-\ell) \mathrm{d} \ell
$$

one gets the reduction and the reduced dynamics

$$
\begin{aligned}
& \eta(t)=x(t)+\int_{t-\tau}^{t} \int_{t-s}^{\tau} e^{(t-s-\ell) A} B(\ell) u(s) \mathrm{d} \ell \mathrm{d} s \\
& \dot{\eta}(t)=(J-R) \nabla H(\eta(t))+\int_{t-\tau}^{\tau} e^{-\ell A} B(\ell) \mathrm{d} \ell u(t) .
\end{aligned}
$$

Remark 3.6: Note that the proof of Lemma 3.1 easily extends the aforementioned classes of time-delay systems. As a matter of fact, in case of one discrete and uniform delay, such a proof might be simplified as follows: because $\eta(t)=e^{-\tau A} x(t+\tau)$ and $x_{\star}$ is an admissible equilibrium assigned by a constant control $u_{\star}$, one easily gets $\eta(t)=$ $e^{-\tau A} x(t+\tau)=e^{-\tau A} x_{\star}=\eta_{\star}$.

\section{A Simulated EXAMPle}

For illustrating the result we consider a simple mass-spring LTI mechanical system of the form

$$
\left(\begin{array}{c}
\dot{q}(t) \\
\dot{p}(t)
\end{array}\right)=\left(\begin{array}{cc}
0 & 1 \\
-1 & -r
\end{array}\right) \nabla H(q(t), p(t))+\left(\begin{array}{l}
0 \\
1
\end{array}\right) u(t-\tau)
$$

with Hamiltonian $H(q, p)=\frac{1}{2 M} p^{2}+\frac{1}{2} K q^{2}$, mass $M>$ 0 , stiffness $K>0$ and $q, p \in \mathbb{R}$ are the position and the momentum of the mass. One computes the matrices

$$
J=\left(\begin{array}{cc}
0 & 1 \\
-1 & 0
\end{array}\right), R=\left(\begin{array}{cc}
0 & 0 \\
0 & r
\end{array}\right), P=\left(\begin{array}{cc}
K & 0 \\
0 & \frac{1}{M}
\end{array}\right) .
$$

Let $x=(q p)^{\top}$ and $x_{\star}=\left(q_{\star} 0\right)^{\top}$ be the desired equilibrium to stabilize via IDA-PBC and reduction.
1) The delay-free design: When $\tau=0$, standard delayfree IDA-PBC applies with $B^{\perp}=\left(\begin{array}{ll}1 & 0\end{array}\right)$ and setting $H_{d}(q, p)=\frac{1}{2 M} p^{2}+\frac{1}{2} K\left(q-q_{\star}\right), P_{d}=P, J_{d}=J$ and

$$
R_{d}=\left(\begin{array}{cc}
0 & 0 \\
0 & r+\kappa
\end{array}\right), \quad \kappa>0 .
$$

Accordingly, the feedback

$$
u_{\mathrm{df}}(t)=-\frac{\kappa}{M} p(t)+K q_{\star}
$$

asymptotically stabilizes the closed-loop equilibrium.

2) Reduction-based design: As $\tau>0$, setting $\eta=$ $\left(\eta_{q} \eta_{p}\right) \in \mathbb{R}^{2}, \alpha=-r / M, \omega=-\alpha \sqrt{1-4 \omega_{n}^{2} / \alpha^{2}}$, $\omega_{n}=\sqrt{K / M}$ and computing

$$
e^{-\tau A}=e^{-\alpha \tau}\left(\begin{array}{cc}
\cos (\omega \tau)+\frac{\alpha}{\omega} \sin (\omega \tau) & -\frac{1}{\omega M} \sin (\omega \tau) \\
M \frac{\omega_{n}^{2}}{\omega} \sin (\omega \tau) & \cos (\omega \tau)-\frac{\alpha}{\omega} \sin (\omega \tau)
\end{array}\right)
$$

the reduced model gets the form of (9) with

$$
B_{\tau}=e^{-\alpha \tau}\left(\begin{array}{c}
-\frac{1}{\omega M} \sin (\omega \tau) \\
\cos (\omega \tau)-\frac{\alpha}{\omega} \sin (\omega \tau)
\end{array}\right) .
$$

At this point, the result in Theorem (3.1) applies by setting

$$
\begin{aligned}
\eta_{\star} & =\left(\begin{array}{l}
\eta_{q_{\star}} \\
\eta_{p_{\star}}
\end{array}\right)=q_{\star} e^{-\alpha \tau}\left(\begin{array}{c}
\cos (\omega \tau)+\frac{\alpha}{\omega} \sin (\omega \tau) \\
M \frac{\omega_{n}^{2}}{\omega} \sin (\omega \tau)
\end{array}\right) \\
B_{\tau}^{\perp} & =e^{\alpha \tau}\left(\frac{\alpha}{\omega} \sin (\omega \tau)-\cos (\omega \tau) \quad \frac{1}{\omega M} \sin (\omega \tau)\right)
\end{aligned}
$$

and $\beta_{\tau}=\frac{1+\alpha^{2} M^{2}}{\omega^{2} M^{2}} \sin ^{2}(\omega \tau)+\cos ^{2}(\omega \tau)-\frac{2 \alpha}{\omega} \sin (\omega \tau) \cos (\omega \tau)$, the feedback law

$$
\begin{aligned}
u_{r}(t)= & -B_{\tau}^{\dagger} R_{d} P \eta(t)-B_{\tau}^{\dagger}\left(J-R_{d}\right) P \eta_{\star}+v(t) \\
= & -\frac{\kappa e^{\alpha \tau}}{\beta_{\tau} M}\left[\cos (\omega \tau)-\frac{\alpha}{\omega} \sin (\omega \tau)\right] \eta_{p}(t) \\
& +\frac{q_{\star}}{\beta_{\tau}}\left(K \cos ^{2}(\omega \tau)-\frac{1}{\omega^{2}}\left(K \alpha^{2}+(r\right.\right. \\
& \left.+\kappa \omega_{n}^{2}-\frac{\omega_{n}}{M}\right) \sin ^{2}(\omega \tau)-\left((r+\kappa) \omega_{n}^{2}\right. \\
& -2 K \alpha) \sin (\omega \tau) \cos (\omega \tau))+v(t) .
\end{aligned}
$$

3) Simulations: We compare the proposed reductionbased control with standard prediction (as discussed in Remark 3.1 over (17). Also, the delay-free closed-loop system under the standard IDA-PBC (18) is reported for completeness. In all cases, we fix $x_{0}=\left(q_{0} p_{0}\right)^{\top}=(-20-$ $1)^{\top}, u(t)=0$ as $t \in[-\tau, 0)$, the desired equilibrium $x_{\star}=\left(\begin{array}{ll}20 & 0\end{array}\right)^{\top}, m=1, K=4, r=0.1$, and $\kappa=1$. Two scenarios are considered: $(i)$ the delay is exactly known (Figure 1); $(i i)$ the delay is small although uncertain (Figure 2) with $\tau \neq \tau_{n}$. In the latter case, as the delay is unknown, the prediction-based feedback cannot be exactly initialized while this problem is overcome by reduction.

In case $(i)$, both controllers get to successfully stabilize the desired equilibrium after a small transient for $t \in[0, \tau)$ in which the control is zero. However, in this particular case performances ensured by the reduction-based IDA-PBC control are significantly improved even with an acceptable control effort. Also, the reduced-dynamics clearly shows that the equilibrium to be stabilized for the reduction is shifted as 

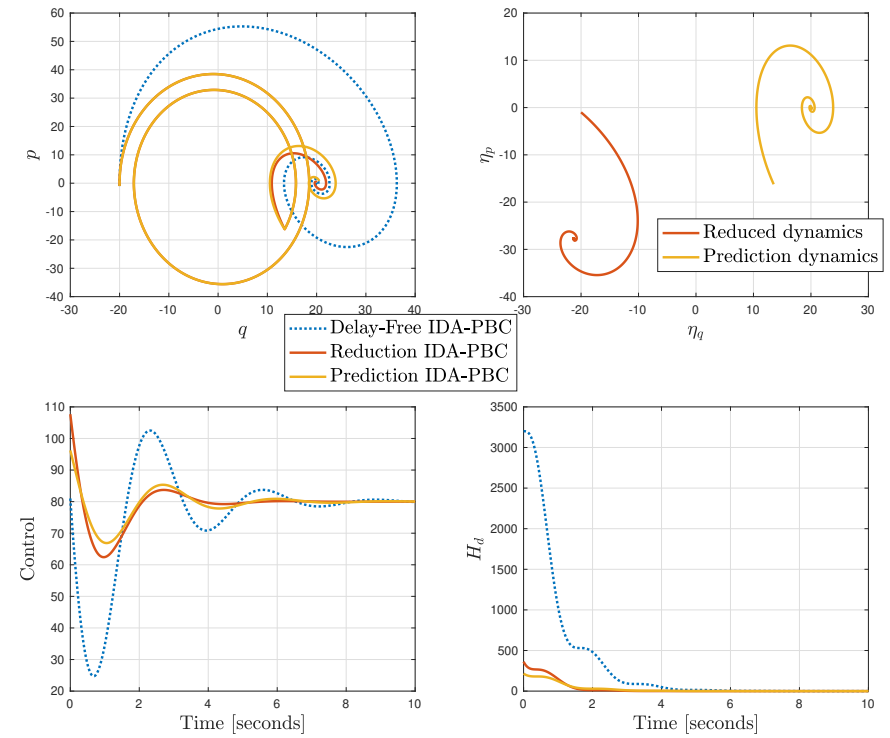

Fig. 1. Known known delay $\tau=\tau_{n}=5 \mathrm{~s}$.
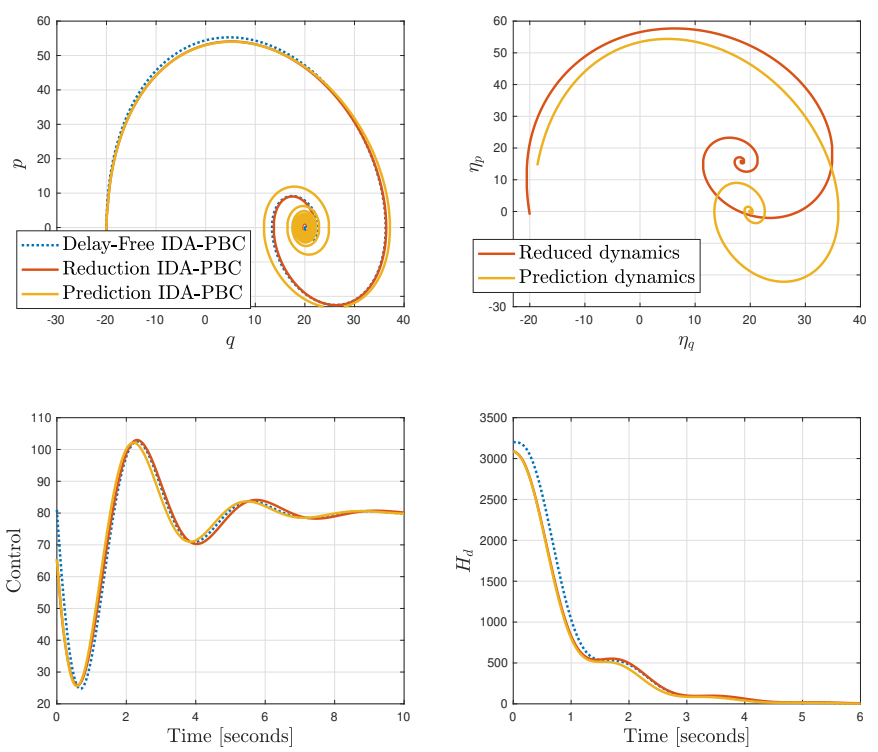

Fig. 2. Small uncertain delay with $\tau=0.15 \mathrm{~s}$ and $\tau_{n}=0.2 \mathrm{~s}$.

proved in Lemma 3.1. We underline that further simulations show that, as $\tau$ is small, performances under both prediction and reduction are similar and satisfying. In case (ii), the reduction-based IDA-PBC control (computed at the nominal $\tau_{n}$ ) stabilizes the desired equilibrium despite the delay mismatch with acceptable performances as well. However, in this same scenario and even under small delay, prediction fails to stabilize the required equilibrium. As a matter of fact, as shown in Figure 2 the controlled dynamics does not converge to the desired $x_{\star}$ under prediction highlighting the improved robustness ensured by reduction.

\section{CONCLUSiOnS}

The problem of regulating the state of linear portcontrolled Hamiltonian systems under input-delays via IDA-
PBC has been considered. Assuming the problem solvable in case of no delay, an IDA-PBC feedback has been constructed based on reduction. Future works concern the extension of this approach to nonlinear Hamiltonian systems, possibly, under sampling using the concepts introduced in [22], [23].

\section{REFERENCES}

[1] A. J. van der Schaft, L2-gain and passivity techniques in nonlinear control. Springer, 2000, vol. 2.

[2] R. Ortega, J. A. L. Perez, P. J. Nicklasson, and H. J. Sira-Ramirez, Passivity-based control of Euler-Lagrange systems: mechanical, electrical and electromechanical applications. Springer Science \& Business Media, 2013.

[3] B. Brogliato, R. Lozano, B. Maschke, and O. Egeland, "Dissipative systems analysis and control," Theory and Applications, vol. 2, 2007.

[4] B. M. Maschke and A. J. van der Schaft, "Port-controlled Hamiltonian systems: modelling origins and system theoretic properties," IFAC Proceedings Volumes, vol. 25, no. 13, pp. 359-365, 1992.

[5] A. van der Schaft and D. Jeltsema, "Port-Hamiltonian systems theory: An introductory overview," Foundations and Trends ${ }^{\circledR}$ in Systems and Control, vol. 1, no. 2-3, pp. 173-378, 2014.

[6] R. Ortega, A. van der Schaft, B. Maschke, and G. Escobar, "Interconnection and damping assignment passivity-based control of portcontrolled Hamiltonian systems," Automatica, vol. 38, no. 4, pp. 585596, 2002.

[7] R. Ortega, A. J. van der Schaft, I. Mareels, and B. Maschke, "Putting energy back in control," IEEE Control Systems Magazine, vol. 21, no. 2, pp. 18-33, 2001.

[8] A. van der Schaft, "Port-hamiltonian modeling for control," Annual Review of Control, Robotics, and Autonomous Systems, vol. 3, pp. 393-416, 2020.

[9] S.-I. Niculescu and R. Lozano, "On the passivity of linear delay systems," IEEE Transactions on Automatic Control, vol. 46, no. 3, pp. 460-464, 2001.

[10] E. Fridman and U. Shaked, "On delay-dependent passivity," IEEE Transactions on Automatic Control, vol. 47, no. 4, pp. 664-669, 2002.

[11] Z. Li, J. Wang, and H. Shao, "Delay-dependent dissipative control for linear time-delay systems," Journal of the Franklin Institute, vol. 339, no. 6-7, pp. 529-542, 2002.

[12] M. S. Mahmoud and A. Ismail, "Passivity and passification of timedelay systems," Journal of Mathematical Analysis and Applications, vol. 292, no. 1, pp. 247-258, 2004.

[13] N. Chopra, "Passivity results for interconnected systems with time delay," in 2008 47th IEEE Conference on Decision and Control. IEEE, 2008, pp. 4620-4625.

[14] M. Mattioni, S. Monaco, and D. Normand-Cyrot, in IEEE Conference on Decision and Control (CDC).

[15] F. Mazenc and D. Normand-Cyrot, "Reduction model approach for linear systems with sampled delayed inputs," IEEE Transactions on Automatic Control, vol. 58(5), pp. 1263 - 1268, 2013.

[16] F. Mazenc and M. Malisoff, "Local stabilization of nonlinear systems through the reduction model approach," IEEE Transactions on Automatic Control, vol. 59, no. 11, pp. 3033-3039, Nov 2014.

[17] F. Mazenc, M. Malisoff, and S. I. Niculescu, "Reduction model approach for linear time-varying systems with delays," IEEE Transactions on Automatic Control, vol. 59, no. 8, pp. 2068-2082, Aug 2014.

[18] Z. Artstein, "Linear systems with delayed controls: a reduction," IEEE Transactions on Automatic control, vol. 27, no. 4, pp. 869-879, 1982.

[19] R. Ortega, Z. Liu, and H. Su, "Control via interconnection and damping assignment of linear time-invariant systems: a tutorial," International Journal of Control, vol. 85, no. 5, pp. 603-611, 2012.

[20] I. Karafyllis and M. Krstic, "Delay-robustness of linear predictor feedback without restriction on delay rate," Automatica, vol. 49, no. 6 , pp. 1761-1767, 2013.

[21] E. Fridman, "Introduction to time-delay systems analysis and control."

[22] M. Mattioni, S. Monaco, and D. Normand-Cyrot, "Sampled-data reduction of nonlinear input-delayed dynamics," IEEE Control Systems Letters, vol. 1, no. 1, pp. 116-121, July 2017.

[23] — " "Nonlinear discrete-time systems with delayed control: A reduction," Systems \& Control Letters, vol. 114, pp. 31-37, 2018. 